\title{
-Short Communications-
}

\section{Use of lectin-FITC conjugates for observation of elastic fibers}

\author{
Tadashi Gotoh and Kazuo Yamashita \\ Department of Anatomy, Nippon Medical School
}

Recently, the roles of elastic fibers. in the subendothelium of blood vessels and the subepithelium of salivary glands have been studied ${ }^{1,2}$. Gotoh, one of the present authors, suggested that elastic fibers play a significant role in preventing distension of the entire heart valve apparatus and in maintaining the internal shape of the heart chamber ${ }^{3)}$. These elastic fibers are fine and rich in microfibrils. There are many methods for staining elastic fibers. These methods provide much information regarding elastin, but very little information regarding microfibrils which are another component of elastic fibers. In addition, to understand their function, it is necessory to survey the three-dimensional features of the structure of these fine fibers. Thus, we require a method for staining microfibrils for further studies of the heart interstitium. It has been reported that these microfibrils consist of fibrillin, a glycoprotein ${ }^{4}$, so in this study we tried to detect three-dimensional features of microfibrilrich elastic fibers by lectin-FITC conjugates under fluorescent microscopy.

The materials used in this study were heart walls and tail tendons of Sprague-Dawley or Wistar-Imamichi rats. To reduce the number of experimental animals killed, we did not use animals only for this experiment, and instead took samples from normal animals that were used for other experiments. Dissected fresh tissues were fixed with $10 \%$ formalin for 10 minutes. After being washed with $0.1 \mathrm{M}$ phosphate buffer, the heart walls were immersed in 5\% Triton X-100 in phosphate buffer for one hour, which removed the epithelial cells of endocardium or epicardium. Tendon collagen bundles were expanded on a glass slide with a needle. After being rinsed with phosphate buffer, these specimens were incubated with FITC labeled-lectin $(5 \mu \mathrm{g} / \mathrm{m} l$, EY Laboratories, Inc.) for one hour at room temperature and observed by fluorescent microscopy after being washed in distilled water.

The lectins used in this experiment and the results summarized in Table 1. Wheat germ agglutinin (WGA) showed the strongest reaction with the elastic fibers. Other lectins (GS-II, $\mathrm{BPA}$, and DBA) showed weaker reactions. However, it was difficult to observed the reactions because of the rapid fade-out of fluorescence. Fig. Ia shows subepithelial connective tissue of the epicardium revealed by WGA-FITC conjugate. The fine networks of elastic fibers can be seen clearly in this figure. The network structure of the subepithelial elastic fibers was confirmed by electron microscopic observation. The three-dimensional image seen in Fig. 1a is not obtained from usual stainings for light microscopic observation. The merit 
Table 1 Reactivity of FITC-conjugated lectins for elastic fibers

\begin{tabular}{|c|c|c|c|c|c|c|c|c|c|c|}
\hline \multirow{2}{*}{ Lectins } & \multicolumn{10}{|c|}{ Carbohydrate Binding Specificity } \\
\hline & $\alpha$ Man & $\alpha \mathrm{Glc}$ & $\alpha \mathrm{GlcNAc}$ & $\beta \mathrm{GlcNAc}$ & $\alpha \mathrm{Gal}$ & $\beta \mathrm{Gal}$ & $\alpha$ GalNAc & $\beta \mathrm{GalNAc}$ & $\alpha$ Fucose & $\alpha$ NeuAc \\
\hline Jackbean (ConA) & $-^{*}$ & - & - & & & & & & & \\
\hline GS-II & & & + & + & & & & & & \\
\hline Wheat $\operatorname{germ}(\mathrm{WGA})$ & & & & H & & & & & & H \\
\hline GS-I & & & & & - & & & & & \\
\hline Osage orange(MPA) & & & & & - & & - & & & \\
\hline Soybean (SBA) & & & & & & & - & & & \\
\hline Peanut (PNA) & & & & & & - & & & & \\
\hline Horse gram(DBA) & & & & & & & + & & & \\
\hline Camel's foot tree(BPA) & & & & & & & + & + & & \\
\hline Grose(UEA-I) & & & & & & & & & - & \\
\hline
\end{tabular}

*- : not detected, + : slight, + : mild, + : intense
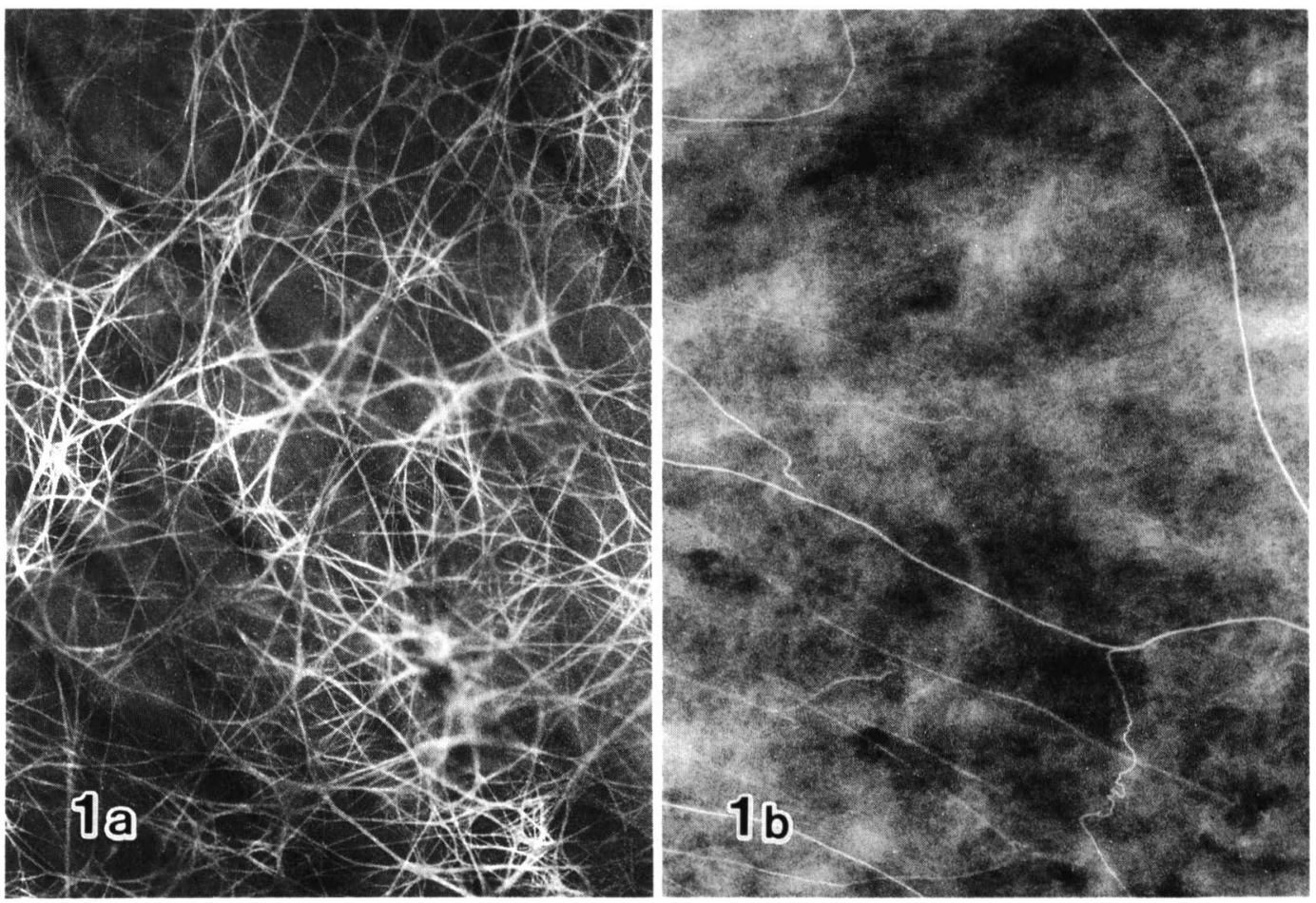

Fig. 1 Fluorescence micrographs showing elastic fibers of the epicardium (a) and the tail tendon (b) treated with WAG-FITC. The array of cardiac muscles can be seen slightly under the network of the elastic fibers (a). Collagen fibrils of torn tendons look like fog (b). a, $\times 340, b, \times 420$

of this method is clear. It has been reported that WGA binds specifically to N-acetyl-Dglucosamine and $\mathrm{N}, \mathrm{N}^{\prime}$-acetylchitobiose ${ }^{5)}$. It has been suggested that these sugar residues are present on microfibrils of elastic fiber ${ }^{6)}$. These sugars are also present in the connective tissue of the interstitium. Surely, a weak reaction is recognized on the collagen fibers in this 
study. The fog-like products in Fig. 1b are the matrix covering collagen fibrils ${ }^{7}$. However, the reaction product of collagen fibers was very weak, and faded within one or two minutes during microscopic observation. The reaction between elastic fibers and WGA is very strong and its fluroscence lasts for a long time. At present, it is not clear why the WGA-reaction is very strong and selective for elastic fibers. In practice, we will be able to investigate elastic fibers of many kinds of tissues with this FITC-lectin method, and may be discover a new important role of elastic fibers.

Good results were not obtained in materials fixed for a long time. Fresh material is best for clear observation.

\section{References}

1) Sakai, T., and Kobayashi, N.: Anat. Embryol., 186, 467, 1992.

2) Lorber, M.: Anat. Rec., 234, 335, 1992.

3) Gotoh, T.: J. Electron Microsc., 43, 151, 1994.

4) Sakai, L.Y., Keene, D.R., and Engvall, E.: J. Cell Biol., 103, 2499, 1986.

5) Peters, B.P., Ebisu, S., Goldstein, I.J., and Flashner, M.: Biochem., 18, 5505, 1979.

6) Serafini-Fracassini, A., Ventrella, G., Griffiths, R., and Hinnie, J.: Conn. Tissue Res., 8, 227, 1981.

7) Gotoh, T., and Sugi, Y.: Cell Tissue Res., 240, 529, 1985. 